Boise State University

ScholarWorks

2-7-2007

\title{
A Field Comparison of Fresnel Zone and Ray-Based GPR Attenuation-Difference Tomography for Time-Lapse Imaging of Electrically Anomalous Tracer or Contaminant Plumes
}

Timothy C. Johnson

Boise State University

Partha S. Routh

Boise State University

Warren Barrash

Boise State University

Michael D. Knoll

Boise State University 


\title{
A field comparison of Fresnel zone and ray-based GPR attenuation-difference tomography for time-lapse imaging of electrically anomalous tracer or contaminant plumes
}

\author{
Timothy C. Johnson ${ }^{1}$, Partha S. Routh ${ }^{1}$, Warren Barrash ${ }^{1}$, and Michael D. Knoll ${ }^{1}$
}

\begin{abstract}
Ground-penetrating radar (GPR) attenuation-difference tomography is a useful tool for imaging the migration of electrically anomalous tracer or contaminant plumes. Attenuation-difference tomography uses the difference in the trace amplitudes of tomographic data sets collected at different times to image the distribution of bulk-conductivity changes within the medium. The most common approach for computing the tomographic sensitivities uses ray theory, which is well understood and leads to efficient computations. However, ray theory requires the assumption that waves propagate at infinite frequency, and thus sensitivities are distributed along a line between the source and receiver. The infinite-frequency assumption in ray theory leads to a significant loss of resolution (both spatially and in terms of amplitude) of the recovered image. We use scattering theory to approximate the sensitivity of electromagnetic (EM) wave amplitude to changes in bulk conductivity within the medium.
\end{abstract}

\begin{abstract}
These sensitivities occupy the first Fresnel zone, account for the finite frequency nature of propagating EM waves, and are valid when velocity variations within the medium do not cause significant ray bending. We evaluate the scattering theory sensitivities by imaging a bromide tracer plume as it migrates through a coarse alluvial aquifer over two successive days. The scattering theory tomograms display a significant improvement in resolution over the ray-based counterparts, as shown by a direct comparison of the tomograms and also by a comparison of the vertical fluid conductivity distribution measured in a monitoring well, located within the tomographic plane. By improving resolution, the scattering theory sensitivities increase the utility of GPR attenuation-difference tomography for monitoring the movement of electrically anomalous plumes. In addition, the improved accuracy of information gathered through attenuation-difference tomography using scattering theory is a positive step toward future developments in using GPR data to help characterize the distribution of hydrogeologic properties.
\end{abstract}

\section{INTRODUCTION}

Ground-penetrating radar (GPR) attenuation-difference tomography is a relatively new method of geophysical imaging that can provide valuable noninvasive information on the distribution of aquifer properties. The basic physical premise behind GPR attenuation-difference tomography is that the attenuation rate of an electromagnetic (EM) wave is strongly dependent upon the bulk conductivity of the medium through which the wave is propagating. For example, consider the amplitude of two EM waves exited from the same location in space, but at different times (time A and B), recorded at a receiving antenna some distance away. Assume that at time A the wave propagates through natural geologic conditions. Furthermore, as sume that an electrically conductive contaminant (or tracer) has invaded the region between the source and receiver at time B. As described by the physics of EM wave propagation (e.g., Maxwell's equations), the amplitude of the trace collected at time B will be attenuated with respect to the amplitude of the trace collected at time A because of the increase in fluid (and hence bulk) conductivity at time B. The difference between the EM wave amplitudes recorded at time $\mathrm{A}$ and $\mathrm{B}$ is dominantly sensitive to the corresponding change in bulk conductivity caused by the invasion of the conductive fluid. In the attenuation-difference tomography experiment, EM wave traces are recorded with geometrically different source-receiver configurations, at times corresponding to time $\mathrm{A}$ and $\mathrm{B}$ in the previous discussion. The difference between trace amplitudes are the data used to to-

Manuscript received by the Editor May 31, 2006; revised manuscript received September 14, 2006; published online February 7, 2007

${ }^{1}$ Boise State University, Department of Geosciences, Boise, Idaho. E-mail: tcj@cgiss.boisestate.edu; routh@ cgiss.boisestate.edu; wb@cgiss.boisestate.edu; mknoll@cgiss.boisestate.edu.

(C) 2007 Society of Exploration Geophysicists. All rights reserved. 
mographically reconstruct the locations and magnitudes of bulk conductivity differences between time A to B.

Previous works employing GPR attenuation-difference tomography focus primarily upon imaging saline tracer movement in fractured aquifer systems (Ramirez and Lytle, 1986; Niva et al., 1988; Olssen et al., 1992; Lane et al., 1999; Day-Lewis et al., 2002; DayLewis et al., 2003; Day-Lewis et al., 2004; Day-Lewis et al., 2005). In addition to monitoring applications, time-lapse tomographic images of contaminant or tracer plumes can help reveal the distribution of the aquifer properties that govern fluid transport in alluvial aquifers. The utility of GPR attenuation-difference tomography in providing information concerning aquifer properties is related directly to the ability of the tomography to resolve small-scale features of the plume. To date, the most common method of attenuation-difference inversion employs ray theory, which assumes waves propagate at infinite frequency. Under the ray assumption, the sensitivity distribution from source to receiver is expressed as a line integral along the raypath, resulting in a loss of resolution when the frequency at which the actual wave propagation takes place is not infinite (Johnson et al., 2005). Johnson et al. (2005), presented a method of providing more resolved tomographic images of temporal changes in bulk conductivity using Fresnel zone attenuation-difference tomography. The Fresnel zone sensitivity matrix represents the physics of EM wave propagation more accurately than ray theory by accounting for finite frequency wave propagation using scattering theory. In this paper, we build upon the theory of Fresnel zone attenuation-difference tomography by demonstrating the method with a field example and comparing the results to the corresponding ray-based results. Our objective in this paper is to demonstrate how attenuation-difference data are reduced in Fresnel zone attenuation difference tomography, to generate Fresnel zone attenuation difference tomograms using field data sets and compare the results to the corresponding raybased tomograms, and to demonstrate, using field data, that Fresnel zone attenuation-difference tomography provides enhanced resolution of changes in bulk conductivity in comparison to ray-based tomography.

We demonstrate a field application of Fresnel zone attenuationdifference tomography (FADT) by imaging an electrically conductive (bromide tracer) plume as it migrates through the subsurface over two successive days. We also show the corresponding raybased attenuation-difference tomography (RADT) tomograms for comparison. We begin with a brief theoretical review of the differences between FADT and RADT and show how the sensitivity matrices are computed for each. Next, we demonstrate and discuss how the field data (including the determination of background velocity, frequency, first pulse power estimation, and location of cells within the Fresnel zone) are reduced. The inverse problem is solved using the LSQR algorithm (Paige and Saunders, 1982) and noise is estimated using the L-curve method (Hansen, 1992). We discuss the L-curves and the insight they provide concerning the signal-to-noise ratio in GPR attenuation-difference data. Finally, we display the tomograms and compare the results to fluid conductivity measurements collected in a test well within the tomographic plane.

The results of the field example are similar to the results shown in the synthetic example of Johnson et al. (2005). Namely, the FADT tomograms are better resolved than the RADT tomograms, both spatially and numerically, because the FADT sensitivity matrix accounts for finite frequency propagation effects. While the FADT and RADT tomograms display the same general plume locations, differences in the tomograms could lead to significantly different interpre- tations of the aquifer properties that govern the dimensions of the plume. The physical improvement in the representation of wave propagation provided by FADT allows us to better leverage the information in the data and produce more accurate images of the plume, leading to a more accurate understanding of the distribution of aquifer properties that affect contaminant migration.

\section{THEORETICAL REVIEW}

\section{Ray-based attenuation-difference tomography (RADT)}

In ray theory, the equations describing the physics of wave propagation are simplified by assuming that waves propagate at infinite frequency. Under this assumption, the sensitivity of wave amplitudes and arrival times are distributed along lines (or rays) such that wave propagation can be represented by a line integral. For EM wave propagation, electric field amplitudes can be expressed as (Lane et al., 1996; Day-Lewis et al., 2002; Holliger and Bergmann, 2002)

$$
D_{a, i}=D_{0 a, i} \Theta_{s a}\left(\phi_{i}\right) \Theta_{r a}\left(\phi_{i}\right) \frac{e^{-\left(\int_{l_{a}} \alpha_{a}(s) d s_{a}\right)}}{\int_{l_{a}} d s_{a}}
$$

where $l_{a}$ is the raypath length from source to receiver at time $t_{a}, D_{0 a, i}$ is the amplitude of the source at position $i$ and time $t_{a}, \alpha_{a}$ is the attenuation coefficient distribution at time $t_{a}, \Theta_{s a}$ is the source radiation pattern at time $t_{a}, \Theta_{r a}$ is the receiver radiation pattern at time $t_{a}, \phi_{i}$ is the angle between source and receiver with respect to horizontal, and $s$ is the curvilinear abscissa along the raypath at time $t_{a}$. In this study, $D_{a, i}$ is the measure of amplitude given by the first pulse energy of trace $i$ in the absence of the bromide tracer plume. As described by Johnson et al. (2005), the measure of amplitude in ray theory is somewhat arbitrary, which is a direct consequence of the assumption of infinite propagation frequency. Note, however, that the Fresnel zone and ray-based methods are exactly equivalent if the frequency of propagation is infinite, demonstrating that the same measure of amplitude can (and should) be used when comparing the methods. We have chosen to represent amplitude as the energy contained in the first pulse in order to be consistent with the Fresnel zone method, which also uses the first pulse energy as the measure of amplitude/attenuation. A definition of the first pulse energy will be given shortly. The energy of the first pulse of the trace collected in the presence of the plume (i.e., the attenuated trace) at time $t_{b}$ is labeled $D_{b, i}$. If we assume that source amplitudes, radiation patterns and raypaths are independent of time, then the difference between the natural logarithm of $D_{a, i}$ and $D_{b, i}$ can be expressed as

$$
\delta D_{i}=\ln \left(D_{a, i}\right)-\ln \left(D_{b, i}\right)=\sum_{j=1}^{N} \delta \alpha_{j} \Delta r_{i j}
$$

Here, the medium has been discretized into $N$ cells. The parameter $\delta \alpha_{j}=\alpha_{b, j}-\alpha_{a, j}$ is the change in attenuation coefficient at cell $j$ between times $t_{a}$ and $t_{b}, \Delta r_{i j}$ is the distance along raypath $i$ through cell $j$. Note that the time invariance of the raypath implies that there is no velocity change in the medium from time $t_{a}$ to $t_{b}$. This is a valid assumption if the only electrical property is changing significantly, as the plume invades the interwell region, is bulk conductivity, because EM wave velocity is a weak function of bulk conductivity (Jackson, 1999). If the invading plume causes significant changes in dielectric permittivity, and thus velocity, then equation 2 is invalid. In our field experiment, we expect no significant temporal variations in dielec- 
tric permittivity and therefore assume raypaths are invariant in time.

In media where galvanic current losses are negligible, or in lowloss media, the change in attenuation coefficient $(1 / \mathrm{m})$ is related to change in bulk conductivity $(\delta \sigma)$ following the mapping (Jackson, 1999)

$$
\delta \alpha_{j} \approx \frac{\delta \sigma_{j}}{2} \sqrt{\frac{\mu}{\epsilon}}
$$

where $\mu$ is the magnetic permeability and $\epsilon$ is the dielectric permittivity. In this study, we assume $\mu$ is equal to its free space equivalent $\left(\mu_{0}\right)$ and that $\epsilon$ is constant in space. The constant $\epsilon$ assumption requires velocity variations within the medium to be small enough that rays travel in approximately straight lines [note EM wave velocity is primarily a function of $\mu$ and $\epsilon$ (Jackson, 1996)]. Our choice of constant $\epsilon$ is based on the relatively small variations in EM wave velocity at the site where the field test is conducted (based on tomographic velocity estimates, the test site has a mean velocity of approximately $0.085-0.095 \mathrm{~m} / \mathrm{ns}$, corresponding to a mean dielectric constant of 12.4 and 10.0, respectively, with a standard deviation of approximately $3.5 \mathrm{~m} / \mathrm{ns}$ in the saturated zone). Substituting equation 3 into equation 2 gives

$$
\delta \mathrm{D}=\mathbf{J}^{\mathrm{S}} \delta \sigma
$$

in matrix form, where $J_{i j}^{S}=\frac{\Delta r_{i j}}{2} \sqrt{\mu / \epsilon}$ and $\delta D_{i}=\ln \left(D_{a, i}\right)-\ln \left(D_{b, i}\right)$. Equation 4 provides a linear map relating changes in bulk conductivity between times $t_{a}$ and $t_{b}$ to the corresponding change in amplitude of the data. In the straight ray case, we image the tracer by inverting $\mathbf{J}^{\mathbf{S}}$ to determine the distribution of $\delta \sigma$.

\section{Fresnel zone attenuation-difference tomography (FADT)}

A complete description of the theory behind FADT for GPR is given in Johnson et al. (2005). Only a brief review is presented here. In order to review the theory behind FADT, we must first discuss how trace amplitudes are measured. The data are defined by

$$
\delta D_{i}=\ln \left(\int_{t_{s r}}^{t_{f p}} e_{b, i}^{2}(t) d t\right)-\ln \left(\int_{t_{s r}}^{t_{f p}} e_{a, i}^{2}(t) d t\right)
$$

where $e_{a, i}(t)$ and $e_{b, i}(t)$ are the time domain traces recorded at times $t_{a}$ and $t_{b}$. Time $t_{s r}$ is the time required for the wave to travel from the source to the receiver (e.g., the first break time), $t_{f p}$ (the first pulse time) is the time to the first zero crossing after $t_{s r}$. The amplitude of the trace between times $t_{s r}$ and $t_{f p}$ is only sensitive to points in space such that energy scattered from those points arrives within the first half period $(T / 2)$. This region is the first Fresnel volume and includes all points $j$ such that (Cerveny and Soares, 1992)

$$
t_{s j}+t_{r j}-t_{s r} \leq \frac{T}{2},
$$

where $t_{s j}$ is the traveltime from the source to scattering location $j$ and $t_{r j}$ is the traveltime from point $j$ to the receiver. The sensitivity of $\delta D_{i}$ to a small bulk conductivity change at point $j\left(\delta \sigma_{j}\right)$ is approximated by the forward difference operator

$$
J_{i j}^{F}=\frac{\ln \left(\int_{t_{s r}}^{t_{f p}} e_{i}^{2}\left(\sigma_{a}(\mathbf{r})+\delta \sigma_{j}, t\right) d t\right)-\ln \left(\int_{t_{s r}}^{t_{f p}} e_{i}^{2}\left(\sigma_{a}(\mathbf{r}), t\right) d t\right)}{\delta \sigma_{j}},
$$

where $\sigma_{a}(\mathbf{r})$ is the bulk conductivity distribution at time $t_{a}$ and position $\mathbf{r}$. In this study, $\sigma_{a}(\mathbf{r})$ represents the background bulk conductivity distribution in the absence of the bromide tracer. The traces $e_{i}\left(\sigma_{a}(\mathbf{r}), t\right)$ and $e_{i}\left(\sigma_{a}(\mathbf{r})+\delta \sigma_{j}, t\right)$ are computed using scattering theory and a first order Born approximation. We assume a dipole radiation pattern in both the source and receiver antennas when computing the traces. In matrix notation, the Fresnel volume equation relating $\delta D_{i}$ to $\delta \sigma_{j}$ is given by

$$
\delta \mathrm{D}=\mathrm{J}^{F} \delta \sigma .
$$

The most important difference between $\mathbf{J}^{S}$ and $\mathbf{J}^{F}$ is that $\mathbf{J}^{F}$ accounts for finite frequency wave propagation and represents the physics of wave propagation more accurately than $\mathbf{J}^{S}$. This accounting improves both the spatial and numerical resolution of inverse estimates of $\delta \sigma$. Examples of $J_{i j}^{S}$ and $J_{i j}^{F}$ for all cells $j$ are shown in Figure 1 . The total sensitivities are equal in each case, but the ray-based sensitivities are compressed to a line. As shown in Johnson et al. (2005), the ray-based sensitivities are too large, which causes the inverse estimates of $\delta \sigma$ to be underpredicted and incorrectly recovered. The ray approximation also causes a loss in the spatial resolution of $\delta \sigma$ by neglecting sensitive regions adjacent to the ray. Both of these effects will be demonstrated and discussed in subsequent sections.

\section{DATA REDUCTION}

To show how the data are reduced, we begin by considering two traces: a trace collected in the absence of the conductive plume $\left(e_{a, i}(t)\right)$ and the corresponding trace collected in the presence of the conductive plume $\left(e_{b, i}(t)\right)$. Field examples of $e_{a, i}(t)$ and $e_{b, i}(t)$ are shown in Figure 2. An algorithm automatically finds $t_{s r}$ and $t_{f p}$ for a)

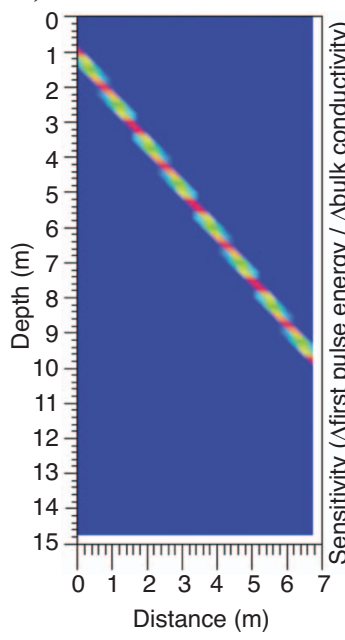

b)



Figure 1. (a) Ray-based attenuation-difference sensitivity distribution and (b) Fresnel zone attenuation-difference sensitivity distribution. Each distribution is computed on a 0.1 by $0.1 \mathrm{~m}$ grid. Note different scales. 
each trace and determines the log energy difference between the first pulses of each trace by equation 5 , computing the integrals numerically. The wave velocity is approximated by dividing the offset (i.e., the distance between the antenna midpoints) by the time to the peak of the first pulse (Vasco and Majer, 1993). Using the average medium velocity, the Fresnel volume boundary is determined by equation 6. Conductive changes in cells outside of this boundary do not affect the first-pulse amplitude because energy scattered from these points arrives after $t_{f p}$. Next, cells within the Fresnel volume are located and sensitivity values are computed for cells $j$ within the volume by equation 7 . When each sensitivity value has been computed, the algorithm moves to the next source-receiver pair and repeats the process, first computing the background trace for that pair and then computing the sensitivities for cells within the Fresnel volume and placing them in the corresponding row of the sensitivity matrix $\mathbf{J}^{F}$.

\section{INVERSE FORMULATION}

The objective of the inversion is to minimize a model objective function subject to fitting the data given by

$$
\Phi\left(\delta \sigma_{\mathrm{est}}\right)=\left\|\mathbf{W}_{d}\left(\mathbf{J} \delta \sigma_{\mathrm{est}}-\delta \mathbf{D}\right)\right\|^{2}+\beta\left\|\mathbf{W}_{m} \delta \sigma_{\mathrm{est}}\right\|^{2},
$$

where $\mathbf{J}$ is $\mathbf{J}^{S}$ or $\mathbf{J}^{F}$, depending on whether RADT or FADT is being used. The vector $\delta \sigma_{\text {est }}$ is the estimated solution, $\mathbf{W}_{d}$ is the data weighting matrix that contains the reciprocal of the standard deviation of the data. In this paper, $\mathbf{W}_{d}$ is an identity matrix (e.g., all data are weighted equally). $\mathbf{W}_{m}$ is the model weighting matrix used to regularize the inverse solution, and $\beta$ is the trade-off parameter. In this study, $\mathbf{W}_{m}$ is the first spatial derivative operator so that the final solution is flat in regions where the data do not constrain the solution. Taking the derivative of $\Phi\left(\delta \sigma_{\text {est }}\right)$ with respect to $\delta \sigma_{\text {est }}$, equating the results to zero and collecting terms provides the normal system of equations to be solved for $\delta \sigma_{\text {est }}$
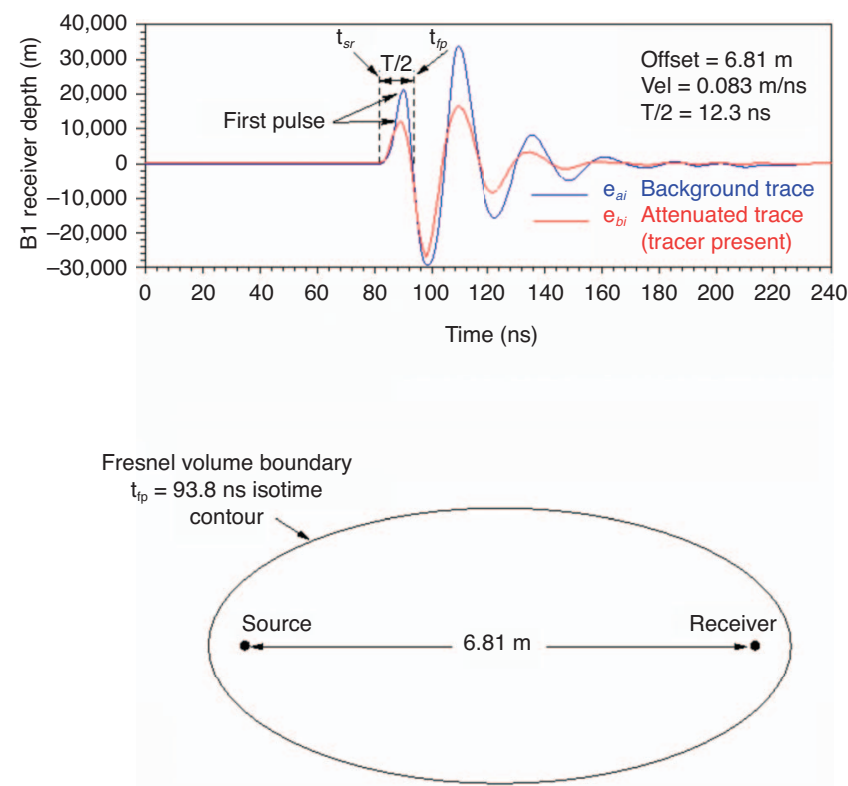

Figure 2. Field examples of a background trace and a trace collected in the presence of a bromide tracer plume and the corresponding Fresnel zone.

$$
\left(\mathbf{J}^{T} \mathbf{W}_{d}^{T} \mathbf{W}_{d} \mathbf{J}+\beta \mathbf{W}_{m}^{T} \mathbf{W}_{m}\right) \delta \sigma_{\mathrm{est}}=\mathbf{J}^{T} \mathbf{W}_{d}^{T} \mathbf{W}_{d} \delta \mathbf{D} .
$$

Instead of solving equation 10 , which requires an expensive matrixmatrix product, we can solve for $\delta \sigma_{\text {est }}$ by minimizing the following system of equations (Paige and Saunders, 1982)

$$
\left[\begin{array}{c}
\mathbf{W}_{d} \mathbf{J} \\
\sqrt{\beta} \mathbf{W}_{m}
\end{array}\right]\left[\delta \sigma_{\mathrm{est}}\right]=\left[\begin{array}{c}
\mathbf{W}_{d} \delta \mathbf{D} \\
0
\end{array}\right] .
$$

Equation 11 only requires a matrix-vector product and can be solved efficiently using the LSQR algorithm (Paige and Saunders, 1982). The appropriate $\beta$ value is chosen by the L-curve (Hansen, 1992) method as described in the next section.

\section{Choosing $\boldsymbol{\beta}$ with the L-curve approach}

In the L-curve approach, the model norm $\left(\Phi_{m}=\left\|\mathbf{W}_{m} \delta \sigma_{\text {est }}\right\|^{2}\right)$ and the data misfit (or data norm) $\left(\Phi_{d}=\left\|\mathbf{W}_{d}\left(\mathbf{J} \delta \sigma_{\text {est }}-\delta \mathbf{D}\right)\right\|^{2}\right)$ are computed for a wide range of $\beta$ values. Then, they are plotted with the model norm on the $\mathrm{x}$-axis and data misfit on the $\mathrm{y}$-axis. The characteristic nature of this curve follows an $\mathrm{L}$ shape. At the maximum point of curvature, a change in $\beta$ influences the model norm and data misfit somewhat equally. To the right of this maximum curvature point, the change in $\beta$ influences the model norm more than data misfit and vice versa. Thus, the $\beta$ obtained in the maximum curvature region of the curve is considered to be the optimal choice and produces a balance between fitting the data and not producing unwanted model structure as indicated by the model norm. In the absence of noise estimates, the L-curve method can provide a good measure of the optimal regularization parameter. We use the L-curve method to estimate the optimal $\beta$ value for both FADT and RADT inverse problems. Then we compare $\delta \sigma_{\text {est }}$ constructed using FADT and RADT with the corresponding optimal $\beta$ values.

\section{TIME LAPSE TRACER TEST AT THE BOISE HYDROGEOPHYSICAL RESEARCH SITE}

The Boise Hydrogeophysical Research Site (BHRS) is an in situ field laboratory located on a gravel bar adjacent to the Boise River about $15 \mathrm{~km}$ southeast of downtown Boise, Idaho (Figure 3). The aquifer at the BHRS consists of coarse (cobble-and-sand) fluvial deposits that overlie a clay layer at approximately $20 \mathrm{~m}$ depth. Eighteen wells were installed at the site in 1997 and 1998 to provide for a wide range of single-well, crosshole, multiwell and multilevel hydrologic, geophysical, and combined hydrologic-geophysical tests (Barrash et al., 1999).

In August 2001, a time-lapse imaging test was conducted at the BHRS to support aquifer characterization and to evaluate the use of GPR for monitoring the transport of electrically conductive fluids (e.g., plumes with high dissolved solids) in heterogeneous granular aquifers (Barrash et al., 2003). A diagram of the inner well field at the BHRS and tracer test configuration is shown in Figure 4. Approximately 1000 gallons of potassium bromide tracer were injected over a period of approximately 30 minutes into a 4-m thick zone that was packed off in well B3. This zone was selected to straddle the contact between a relatively low-porosity layer above $11 \mathrm{~m}$ in well B3, and a higher-porosity layer below $11 \mathrm{~m}$ in well B3 (Figure 4; Barrash and Clemo, 2002).

Over the next 17 days, the tracer migrated along approximately the natural gradient, following a path from well B3 to B6. Wells B1, 
B2, B4, B5, and B6 were instrumented with packer systems that isolated six 1-m zones in the mid-section of each well that overlapped the 4-m injection zone in B3. Each packer system was constructed to allow for the presence of radar antennas during tomographic data collection and each zone was monitored for changes in fluid conductivity throughout the test. A packer system with twenty $25-\mathrm{cm}$ thick monitoring zones was placed in well A1 to capture high-resolution tracer concentration behavior as the plume migrated through the system (Barrash et al., 2002).

\section{Attenuation-difference data}

Tomographic radar data were collected daily over the course of the test in planes B1-B4, B2-B4, and on three separate days in B3B6. For the B1-B4 plane, data were acquired with a $100 \mathrm{MHz}$ Mala Geosciences RAMAC/GPR system at every $20 \mathrm{~cm}$ in the receiver well B1 and every $5 \mathrm{~cm}$ in the source well B4. In this study, we compare the B4-B1 FADT and RADT attenuation-difference tomograms constructed from data collected on the ninth and tenth days of the test, using reference (or background) data collected on the second day of the test. Data collected on the second day are adequate as background data because no significant amounts of tracer were located within any first Fresnel zones by the second day. The inverted data are typically spaced at $20 \mathrm{~cm}$ intervals in each well over a $15 \mathrm{~m}$ interval for a total of 2861 data points. The tomographic grid consists of $25 \times 25 \mathrm{~cm}$ cells for a total of 1586 cells.

Figure 5 shows the B1-B4 attenuation-difference data plots at nine and 10 days after the tracer was injected. The horizontal axis indicates the transmitter depth in B4 and the vertical axis indicates the receiver depth in $\mathrm{B} 1$. The color scale represents the attenuation-difference magnitude of each source-receiver pair as given by equation 5 where the trace $e_{a, i}$ was collected two days after injection and $e_{b, i}$ was collected nine (or 10) days after injection. The low attenuationdifferences below $9 \mathrm{~m}$ and above $15 \mathrm{~m}$ suggest that the plume is

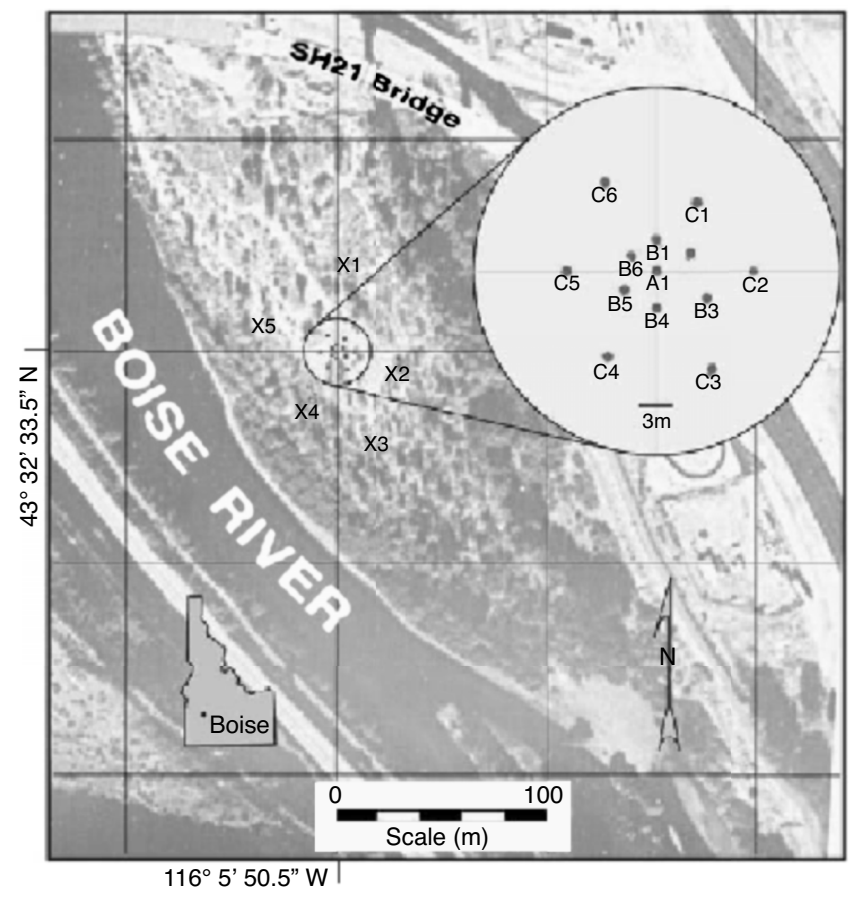

Figure 3. Aerial view of BHRS, Boise River, and well locations. concentrated in the middle portion of the tomographic plane. This is expected because the $4 \mathrm{~m}$ injection interval ranges from 9 to $13 \mathrm{~m}$ depth. Note also that the distribution of attenuation differences in the crossplots are similar for both days, although day 10 displays relatively larger attenuation differences. It is plausible that the general shape of the plume is similar on days nine and 10, but that, in detail, higher general tracer concentrations and larger plume dimensions have moved into the tomographic plane on day 10 , causing the larger

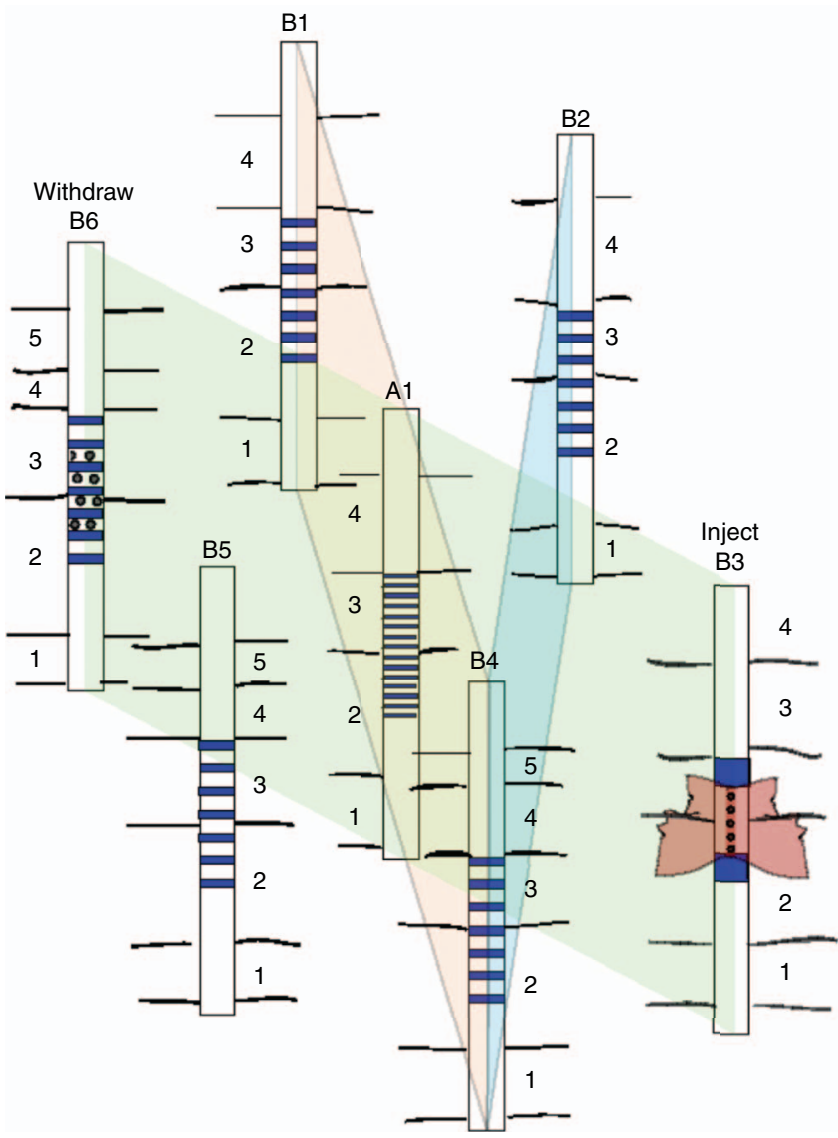

Figure 4. Schematic diagram of the inner well-field at the BHRS showing time-lapse tracer test configuration. The injection zone straddles the contact between the lower porosity zone 3 and the higher porosity zone 2 (Barrash and Clemo, 2002). Over the course of the test, the plume migrates along approximately natural gradient from $\mathrm{B} 3$ through $\mathrm{A} 1$ to $\mathrm{B} 6$. Tomograms shown in this paper are constructed for the B1-B4 plane on the ninth and 10th day after injection. Note the porosity contact locations between zones 2 and 3 in each well, demonstrating the lateral extent of each zone.
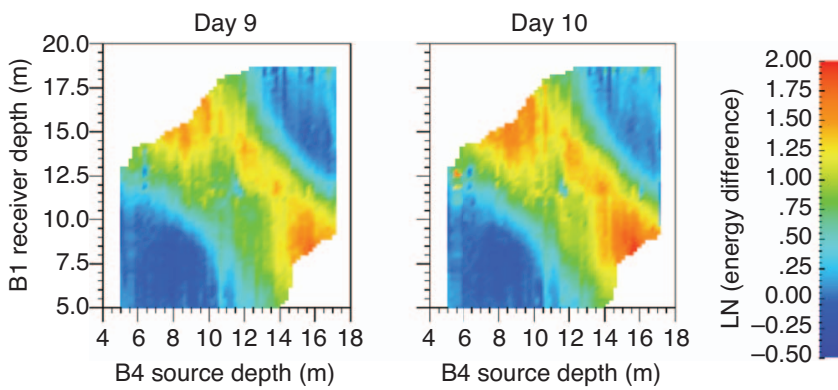

Figure 5. B1-B4 attenuation difference data cross-plots at nine and 10 days after injection 
attenuation differences. Also note the attenuation-difference hole that appears when the transmitter and receiver are located at approximately 12 and $11.5 \mathrm{~m}$ depth, respectively. As we will show, the inverse mapping of this feature is readily observable in the tomograms.

Figure 6 shows the zero-offset profile (or level runs) nine and 10 days after injection. The low first-pulse energy difference at approximately $12-\mathrm{m}$ depth corresponds to the hole in the first pulse energy difference crossplots shown in Figure 5. The zero-offset profiles display separate peaks for both days, indicating that the plume may have two separate lobes above and below approximately $12-\mathrm{m}$ depth. Also note that the attenuated trace shown in Figure 2 was collected with the source and receiver antennas at a 13-m depth in wells B3 (the injection well) and B6 (the withdrawal well), respectively, a)



b)



Figure 6. B4-B1 first-pulse energy difference zero-offset profiles (level runs) (a) nine days and (b) 10 days after injection.
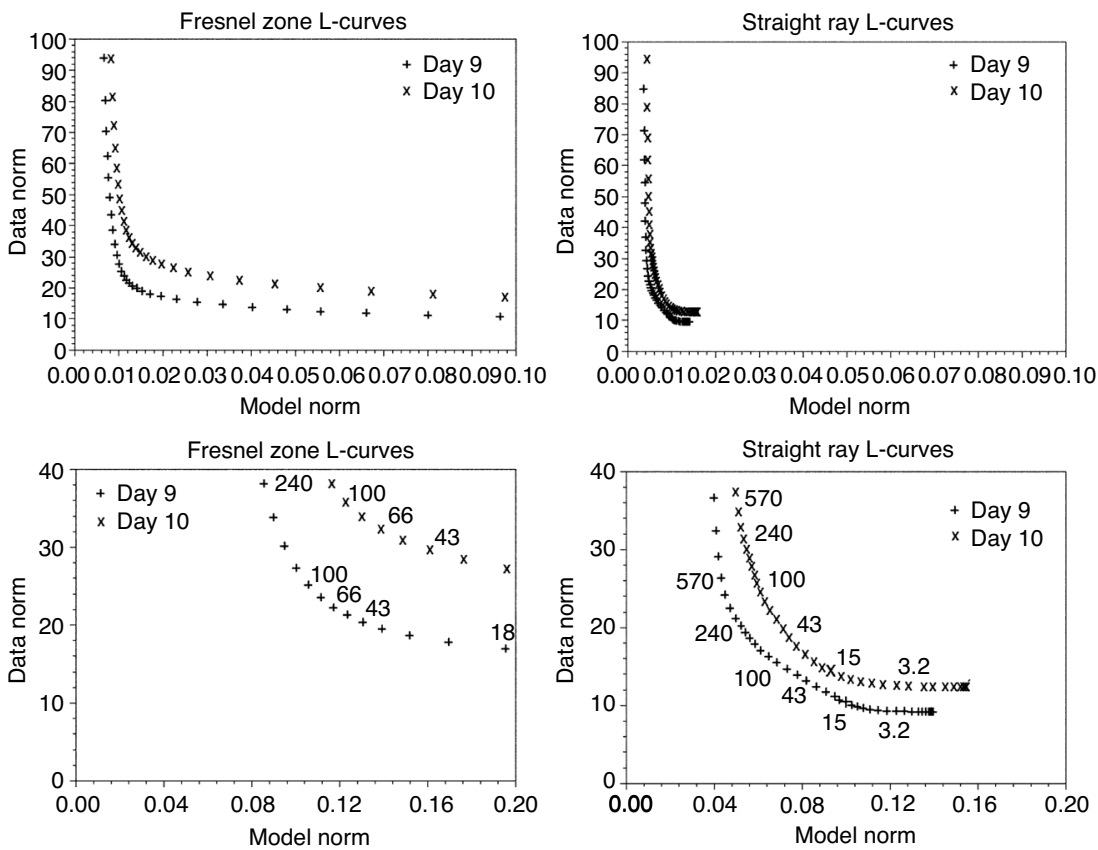

Figure 7. Fresnel zone (left) and straight ray (right) L-curves. The lower row is a magnification of the upper row about the elbow. $\beta$ values are shown in the lower curves. The minimum radius of curvature occurs at approximately $\beta=66$ for the Fresnel zone inversions and $\beta=15$ for the straight ray inversions. on the tenth day of the test. The background trace was collected before the bromide was injected. The large attenuation displayed in this trace is caused by the presence of bromide tracer between well $\mathrm{B} 3$ and $\mathrm{B} 6$ at a 13-m depth.

\section{L-curve analysis}

Figure 7 compares the L-curves for the FADT and RADT inversions. Each point on the L-curve is generated by solving equation 10 for a constant $\beta$ value, and the optimum $\beta$ value occurs at the point of greatest curvature (Hansen, 1992). Comparing the L-curves between days nine and 10 we see that, for a given $\beta$ value, day 10 consistently has a larger data norm than day nine for both the FADT and RADT inversions, suggesting more noise in the day 10 attenuationdifference data. However, the optimum $\beta$ value for each day is the same ( $~ 66$ for FADT, as shown in Figure 7$)$. If the day 10 data have a larger noise component than the day nine data, then the optimal $\beta$ value for day 10 should be larger than the optimal $\beta$ value for day nine. This apparent discrepancy leads to some insight about radar attenuation-difference data that can be explained as follows. Note that with respect to day nine, the day 10 model norm also increases for a given $\beta$ value. This is reasonable because the attenuation-difference values (and hence inverse model values) are greater on day 10 than on day nine, leading to a larger model norm. In other words, we have larger signals (attenuation differences) and a larger noise component (as indicated by the data norm at the optimal $\beta$ value) on day 10 compared to day nine. The constant $\beta$ value for the two days suggests that the signal-to-noise ratio is constant for each day. In fact, although we only show inversions for two days in this paper, the L-curves constructed for day six through day 15 inversions display approximately the same optimal $\beta$ values. The constant optimal $\beta$ value reinforces the compatibility between the data sets, a crucial aspect for timelapse imaging, and suggests the data reduction procedure prior to inversion is able to preserve the changes that are comparable. On day six, for example, the plume is just visible and the optimal $\beta$ value results in a small model and data norm. The constant $\beta$ value for each day suggests that for this test, time-lapse radar attenuation-difference data display the same signal-to-noise ratio and thus the same optimal $\beta$ value. This is important because, if optimal $\beta$ values are constant, then the effort required to produce appropriately regularized and comparable time-lapse inversion results will be greatly reduced. This may help increase the utility of time-lapse tomography and facilitate technological advances such as realtime imaging.

Note that the FADT and RADT inversions display significantly different optimal $\beta$ values, data norms, and model norms. These differences arise because of the difference in operators mapping the model to the data (i.e., $\mathbf{J}^{\mathrm{F}}$ versus $\mathbf{J}^{\mathrm{S}}$ ). The SVD analysis conducted in Johnson et al. (2005) shows that RADT requires more basis functions than FADT to reconstruct a model to the same noise level. Including more basis functions is analogous to reducing the $\beta$ value. Thus, we observe that the optimal $\beta$ value is lower for RADT in comparison to FADT. In addition, the higher or- 
der basis functions for RADT saturate such that including more basis functions does not change the model norm significantly. This effect is illustrated by the RADT L-curves in Figure 7. For example, given a data norm of 10 we see that the model norm for FADT is an order of magnitude greater than the model norm for RADT.

\section{Attenuation-difference tomograms}

The FADT and RADT attenuation-difference tomograms for days nine and 10 are compared in Figure 8. The tomograms are oriented such that the injection well B3 is behind the page and the extraction well B6 is in front of the page. Wells B1 and B4 are on the left and right boundaries of the tomograms, respectively, and the plume is migrating out of the page toward the reader. The test was configured so that the injection interval straddled an approximately horizontal boundary located at approximately 11-m depth in well B3 that separates a relatively low-porosity zone (Figure 4, zone 3) from a higher porosity zone (Figure 4, zone 2 ) that is persistent throughout the test region (Barrash and Clemo, 2002). Each of the tomograms indicate that on days nine and 10 , most of the plume is located in the high porosity zone below $12 \mathrm{~m}$ in the B1-B4 plane. The FADT tomograms suggest that the plume is divided into an upper lobe and a lower lobe. The lobes are smeared together in the RADT tomograms suggesting the plume is connected across the porosity boundary.

The smearing between lobes in the RADT case is a consequence of the ray approximation. For example, consider the trace collected on day nine with the source and receiver both at $12 \mathrm{~m}$ depth in their respective wells. If we assume the plume consists of an upper and a lower lobe separated by approximately $1.5 \mathrm{~m}$, then the trace will be attenuated with respect to the background trace because much of the plume in each lobe is located within the first Fresnel zone. Thus, the datum associated with the source and receiver displays attenuation.

Now consider the ray associated with the source-receiver pair. The ray-based sensitivities are located along a line between the source and receiver and do not represent sensitive regions adjacent to the ray that are caused by finite frequency propagation. In order to fit the datum the inversion routine must smear the boundaries of the plume to the ray, thereby resulting in a loss of spatial resolution. This can explain why the lobes appear connected in the RADT inversion but separated in the FADT inversion. The ray-based inversion is forced to smear the boundaries of the plume in order to fit data points associated with rays passing near the plume boundaries. Note also that the predicted bulk conductivity changes are significantly lower in the ray-based tomograms. This occurs because sensitivities are concentrated along the ray and overpredict the sensitivity of each cell within the ray, resulting in a poor amplitude recovery. The loss of spatial reso- lution and poor amplitude recovery for RADT were also shown for a synthetic case by Johnson et al. (2005).

\section{Comparison with fluid conductivity}

Comparisons of normalized predicted bulk conductivity versus normalized measured fluid conductivity at well A1 are shown in Figure 9. It is important to interpret these comparisons in context. The support volumes for the fluid conductivity measurements are essentially one-dimensional point values aligned at 25 -cm intervals along A1. In contrast, the radar support volume (i.e., the Fresnel volume) is a 3D volume on the order of several cubic meters (Figure 2). The plume will be sensed during radar propagation and will be located in the tomograms at the point where it reaches the Fresnel volume boundary, but before it reaches A1, thereby resulting in a discrepancy (or time lag) between radar and fluid conductivity measurements made at a given time. In the FADT case, this data discrepancy is a
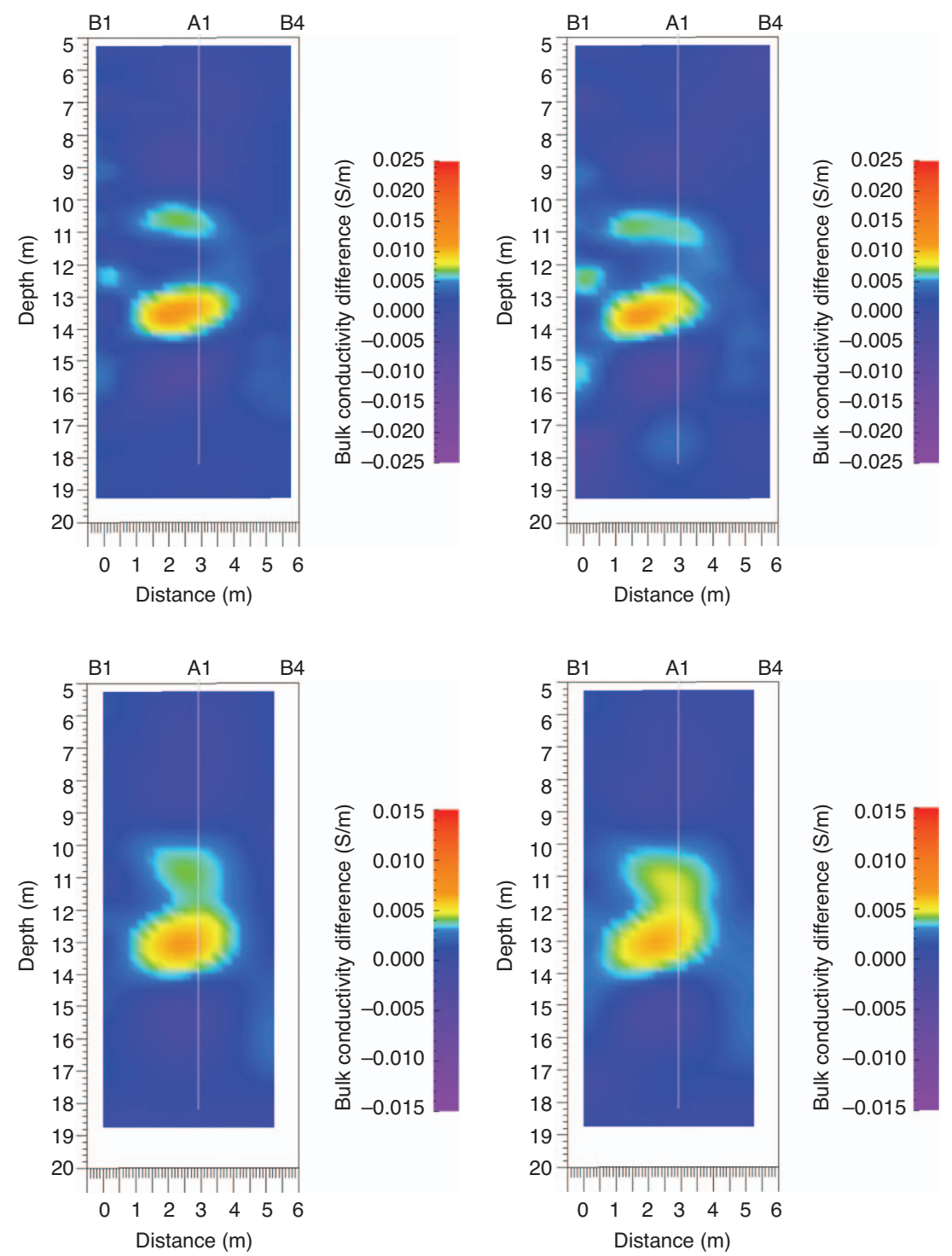

Figure 8. (top row) B4-B1 FADT and (bottom row) RADT tomograms at (left column) nine and (right column) 10 days after injection. 
consequence of the 2D inversion. If a 3D inversion was conducted, there would be no discrepancy between the attenuation difference and fluid conductivity data because the FADT method would account for out-of-plane sensitivities. Although we have conducted the 2D inversion in this case (and therefore the data discrepancy exists), it is useful to qualitatively compare the fluid conductivity measurements to the inverse estimates at A1. For instance, the upper lobe shown in the tomograms does not appear in the fluid conductivity measurements. This suggests that the upper lobe has not yet reached A1 but is within the Fresnel volume of the radar data. Thus, the lower

a)

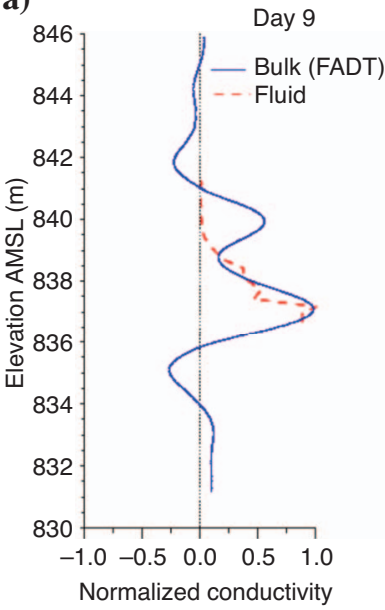

d)



b)

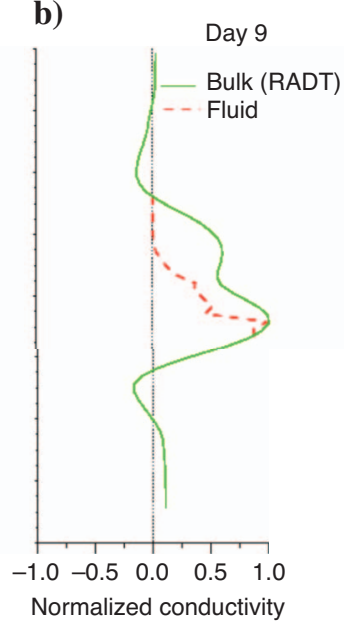

e)



lobe (which has reached A1) is moving faster than the upper lobe, which is consistent with the porosity structure of the BHRS (assuming that the ratio of hydraulic conductivity to porosity is positively correlated with porosity at the BHRS). The fluid conductivity measurements are also consistent with the tomographic structure of the lower lobe. The conductivity peaks are colocated and the vertical extent of the predicted bulk conductivity change matches well with the measured fluid conductivity change, at least to the depth where fluid conductivity measurement are available. This is most evident in the FADT inversions, which are less affected by smearing artifacts.

The negative portions of the tomograms (which are somewhat hidden by the color scale in Figure 8) are most likely tomographic artifacts. We assume the negative lobes are artifacts because negative bulk conductivity changes are not sensible given the nature of the tracer test (e.g., all bulk conductivity changes should be positive). In addition, fluid conductivity measurements in the monitoring wells indicate no negative changes in fluid conductivity with respect to pretest levels. The negative artifacts could possibly be removed with a more advanced inversion technique (e.g., positivity constraints), but no such inversion was attempted in this work.

\section{CONCLUSIONS}

$0.0 \quad 005.010 .015$
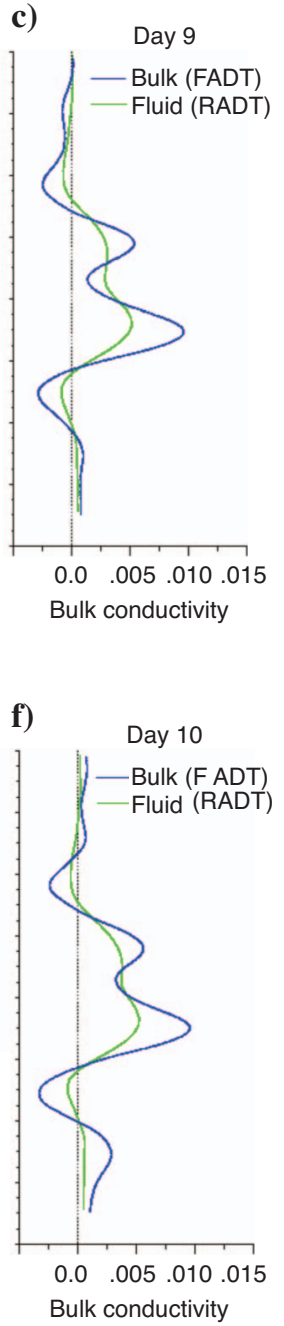

We have shown a practical application of attenuation-difference tomography with the intent of demonstrating the utility of Fresnel zone tomography versus traditional ray-based methods. Although the tomographic images are interesting on their own, they are not of much value unless they can be used to give greater insight into the distribution of subsurface properties, or otherwise solve some problem. Attenuation-difference tomography is a useful tool for monitoring the migration of conductively anomalous fluids through the subsurface. However, in the current state of the practice, using the tomographic images to infer something about subsurface properties requires some type of interpretation, whether mathematical or in the form of expert judgment. For instance, we may wish to use the tomograms to estimate the actual boundaries of the plume in the tomographic plane, which are dependent

Figure 9. (a) Normalized FADT bulk conductivity and measured fluid conductivity in well A1 nine days after injection. (b) Normalized RADT bulk conductivity and measured fluid conductivity in well A1 nine days after injection. (c) FADT and RADT bulk conductivity at well A1 nine days after injection. (d) Normalized FADT bulk conductivity and measured fluid conductivity in well A1 10 days after injection. (e) Normalized RADT bulk conductivity and measured fluid conductivity in well A1 10 days after injection. (f) FADT and RADT bulk conductivity at well A1 10 days after injection. The fluid conductivity comparisons demonstrate the smearing effect of RADT. (b) and (e) The lower lobe of the plume in the RADT profiles has a larger vertical extent than is suggested by the fluid conductivity measurements. (c) and (f) demonstrate the magnitudes of the FADT versus RADT bulk conductivity change at well A1. The RADT values are low because of the erroneously high sensitivities along the ray. The upper lobe of the plume is not shown in the fluid conductivity measurements because it has not reached A1, but is within the sampling volume (i.e., the Fresnel volume) of the GPR data, and is thus mapped to the tomographic plane. Bulk conductivity upon lateral and vertical hydraulic dispersivities, or we may wish to generate pseudobreak through curves based upon time lapse tomographic images for use in hydrogeologic parameter estimation (which would require a bulk conductivity to fluid conductivity petrophysical transform). In any case, the accuracy of the interpretation and conclusions based on the interpretation is dependent upon the accuracy of the tomogram(s).

By better representing the physics of wave propagation in attenuation-difference tomography, we can better leverage the information content in the radar data to produce more accurate estimates of the distribution of fluid conductivity 
anomalies. By producing more accurate tomograms, we can reduce the possibility of interpretational errors. The field example presented in this paper is illustrative in this regard. Although the Fresnel zone and ray-based tomograms display the same general distribution of conductivity changes, the Fresnel zone images suggest the plume consists of two relatively compact lobes while the ray-based images suggests the plume is more continuous and extensive. These two images could lead to significantly different inferences about the subsurface and the distribution of aquifer properties. For example, we may conclude, based on the FADT tomograms, that there is a low hydraulic conductivity wedge centered at approximately a $12-\mathrm{m}$ depth causing the separation of the plume into an upper and lower lobe. The RADT tomograms do not suggest such a feature because the lobes are more continuous, which may lead to the interpretation of a more homogeneous subsurface. In addition, the RADT tomograms may lead to erroneously large estimates of dispersivity values, because the boundaries of the plume must be extended in order to satisfy the data in the RADT method. Because the FADT images are more accurate, we expect to gain more accurate knowledge about the subsurface and reduce the possibility of false interpretation by employing Fresnel zone tomography.

Ideally, it would be possible to use tomographic GPR attenuationdifference data directly to help calibrate flow and transport models via joint inversion. Such a development would be a significant advancement in hydrogeophysics because tomographic radar data contain a tremendous amount of information concerning the shape plume, and thus the hydrogeologic properties that control the plumes structure. We believe that the development of FADT is an important step toward making possible the joint inversion of hydrogeologic and GPR attenuation-difference data, not only because FADT more accurately resolves plume dimensions, but also because FADT more accurately recovers the magnitude of bulk-conductivity changes than does RADT. Both of these advantages are important to eliminate RADT-based inconsistencies between the attenuation-difference data and hydrogeologic or transport data that may be available. For instance, consider a fluid conductivity measurement taken just above the boundary of a plume, indicating that no tracer is present. The RADT method may suggest tracer is present at that point because the datum feels the effects of the plume, but the ray does not pass directly through the plume, leading to an inconsistency between the measured fluid conductivity and the RADT data. A similar argument could be made concerning the magnitude of measured and RADT predicted fluid conductivity values (assuming a valid bulkconductivity to fluid-conductivity transform were available), because RADT significantly underpredicts bulk conductivity changes. FADT resolves these issues, bringing the possibility of joint inversion to calibrate groundwater models closer to reality.

\section{ACKNOWLEDGMENTS}

We would like to thank Fred Day-Lewis for his thoughtful and thorough review of this work. Two anonymous reviewers also pro- vided helpful comments. Funding for this work was provided by EPA grant X-970085-01-0 and the Inland Northwest Research Alliance (Ph.D. fellowship for Timothy Johnson). Computations were performed on the geophysical computing system (NSF-EPSCoR grant no. EPS0132626) at the Center for Geophysical Investigation of the Shallow Subsurface at Boise State University. We would also like to thank the many people who helped with data collection and logistics during the tracer test.

\section{REFERENCES}

Barrash, W., and T. Clemo, 2002, Hierarchical geostatistics, multifacies systems, and stationarity: Boise Hydrological Research Site, Boise Idaho: Water Resources Research, 38, no. 10, 1196.

Barrash, W., T. Clemo, D. Hyndman, E. Reboulet, and E. Hausrath, 2002, Tracer/time-lapse radar imaging test: Design, operation, and preliminary results, Technical Report BSU CGISS 02-02, Center for the Investigation of the Shallow Subsurface, Boise State University.

Barrash, W., T. Clemo, and M. Knoll, 1999, Boise Hydrogeophysical Research Site (BHRS), objectives, design, initial geostatistical results: Proceedings of the Symposium on the Application of Geophysics to Engineering and Environmental Problems, 389-398.

Barrash, W., M. Knoll, D. Hyndman, T. Clemo, E. Reboulet, and E. Hausrath, 2003, Tracer/time-lapse radar imaging test at the Boise Hydrogeophysical Research Site: Symposium on the Application of Geophysics to Engineering and Environmental Problems, 163-174.

Cerveny, V., and J. Soares, 1992, Fresnel volume ray tracing: Geophysics, $\mathbf{5 7}, 902-915$.

Day-Lewis, F., J. Harris, and S. Gorelick, 2002, Time-lapse inversion of crosswell radar data: Geophysics, 67, 1740-1752.

Day-Lewis, F., J. Lane, and S. Gorelick, 2004, Combined interpretation of radar, hydraulic and tracer data from a fractured rock aquifer: Hydrogeology Journal, 14, no. 1-2, 1-14

Day-Lewis, F., J. Lane, J. Harris, and S. Gorelick, 2003, Time-lapse imaging of saline tracer tests using cross-borehole radar tomography: Water Resources Research, 39, no. 10, 1290-1303.

Day-Lewis, F., K. Singha, and A. Binley, 2005, Applying petrophysical models to radar travel time and electrical resistivity tomograms, Resolution-dependent limitations: Journal of Geophysical Research, 110, no. 80, 206.

Hansen, P., 1992, Analysis of discrete ill-posed problems by means of the L-curve: SIAM, 34, 561-580.

Holliger, K., and T. Bergmann, 2002, Numerical modeling of borehole georadar data: Geophysics, 67, 1249-1257.

Jackson, J., 1999, Classical electrodynamics, 3rd ed.: Wiley \& Sons, Inc.

Johnson, T., P. Routh, and M. Knoll, 2005, Fresnel volume georadar attenuation-difference tomography: Geophysical Journal International, 162, 9-24.

Lane, J., D. Wright, and P. Haeni, 1999, Borehole radar tomography using saline tracer injections to image fluid flow in fractured rock: USGS Toxic Substances Hydrology Meeting.

Niva, B., O. Olssen, and P. Blumlung, 1988, Grimsel test site: Radar crosshole tomography with application to migration of saline tracer through fracture zones: Technical Report TR88-31, Swedish Geological Co.

Olssen, O., L. Falk, O. Forslund, L. Lundmark, and E. Sandberg, 1992, Borehole radar applied to the characterization of hydraulically conductive fracture zones in crystalline rock: Geophysical Prospecting, 40, 109-142.

Paige, C., and M. Saunders, 1982, LSQR: An algorithm for sparse linear equations and sparse least squares: Association for Computing Machinery Transactions on Mathematical Software, 8, 43-71.

Ramirez, A., and R. Lytle, 1986, Investigation of fracture flow paths using alterant geophysical tomography: International Journal of Rock Mechanics and Mining Sciences, Geomechanical Abstracts, 23, no. 2, 165-169.

Vasco, D., and E. Majer, 1993, Wavepath traveltime tomography: Geophysical Journal International, 115, 1055-1069. 